\title{
Kinase switching in mesenchymal-like non-small cell lung cancer lines contributes to EGFR inhibitor resistance through pathway redundancy
}

\author{
Stuart Thomson · Filippo Petti · Izabela Sujka-Kwok • \\ David Epstein · John D. Haley
}

Received: 25 April 2008/Accepted: 22 July 2008/Published online: 12 August 2008

(C) The Author(s) 2008. This article is published with open access at Springerlink.com

\begin{abstract}
NSCLC cells with a mesenchymal phenotype have shown a marked reduction in sensitivity to EGFR inhibitors, though the molecular rationale has remained obscure. Here we find that in mesenchymal-like tumor cells both tyrosine phosphorylation of EGFR, ErbB2, and ErbB3 signaling networks and expression of EGFR family ligands were decreased. While chronic activation of EGFR can promote an EMT-like transition, once having occurred EGFR family signaling was attenuated. We investigated the mechanisms by which mesenchymal-like cells bypass EGFR signaling and acquire alternative routes of proliferative and survival signaling. Mesenchymal-like NSCLC cells exhibit aberrant PDGFR and FGFR expression and autocrine signaling through these receptors can activate the MEK-ERK and PI3K pathways. Selective pharmacological inhibition of PDGFR or FGFR receptor tyrosine kinases reduced cell proliferation in mesenchymal-like but not epithelial NSCLC cell lines. A metastable, reversible EMT-like transition in the NSCLC line H358 was achieved by exogenous $\operatorname{TGF} \beta$, which served as a model EMT system. The H358/TGF $\beta$ cells showed many of the attributes of established mesenchymal-like NSCLC cells including a loss of cell-cell junctions, a loss of EGF-family ligand expression, a loss of ErbB3 expression, increased EGFRindependent Mek-Erk pathway activation and reduced sensitivity to EGFR inhibition. Notably an EMT-dependent acquisition of PDGFR, FGFR and TGF $\beta$ receptors in
\end{abstract}

S. Thomson $(\bowtie) \cdot$ F. Petti · I. Sujka-Kwok · D. Epstein ·

J. D. Haley $(\bowtie)$

Department of Translational Research, OSI Pharmaceuticals

Inc., 1 Bioscience Park Drive, Farmingdale, NY 11735, USA

e-mail: sthomson@osip.com

J. D. Haley

e-mail: jhaley@osip.com
H358/TGFbeta cells was also observed. In H358/TGFbeta cells both PDGFR and FGFR showed functional ligand stimulation of their intrinsic tyrosine kinase activities. The findings of kinase switching and acquired PDGFR and FGFR signaling suggest investigation of new inhibitor combinations to target NSCLC metastases.

Keywords Epithelial mesenchymal transition . EGFR · PDGFR · FGFR - TGF beta · NSCLC

\section{Introduction}

As human cancers progress to a more invasive, metastatic state, altered cell signaling is required to prevent apoptotic and anoikis signals associated with epithelial cell detachment [1]. Recent data highlight the transdifferentiation of epithelial cancer cells to a more mesenchymal-like state, a process resembling epithelial-mesenchymal transition (EMT) [2, 3], to facilitate cell invasion and metastasis [4, 5]. Through EMT-like transitions mesenchymal-like tumor cells are thought to gain migratory capacity at the expense of proliferative potential. A reverse mesenchymal-epithelial transition (MET) has been postulated to regenerate a more proliferative state and allow macrometastases resembling the primary tumor to form at distant sites [6]. The transition of tumor cells at the stromal-tumor interface to a more mesenchymal-like state likely plays a role in the progression of cancer [7, 8] and has been correlated with poor prognosis $[9,10]$. Our previous studies have shown that the cellular changes associated with EMT-like transitions alter the dependence of carcinoma cells on epidermal growth factor receptor (EGFR) signaling networks for proliferation and survival. An EMT-like transition has been associated with NSCLC tumor cell, xenograft and patient insensitivity to 
selective EGFR tyrosine kinase inhibition [11-13], in part from EGFR independent activation of either or both the PI3'kinase or Mek-Erk pathways [14]. Similar data correlating EMT status to sensitivity to EGFR TKIs have been reported in pancreatic, CRC [14] bladder [15] and HNSCC [16] cell lines, xenografts and in patients samples [13]. The molecular determinants to alternative routes of activation of the PI3'kinase and/or Erk pathways, which can bypass cellular sensitivity to EGF receptor inhibitors, have been actively investigated. For example within an epithelial tumor context IGF-1R activation has been associated with insensitivity to EGFR inhibition [17, 18], likely through compensatory IGF-1R-PI3K-Akt signaling rendering EGFR family signaling redundant. Similarly the amplification of the HGF receptor tyrosine kinase, c-Met, has been shown to compensate for EGFR inhibition in part through recruitment of ErbB3 to activate the PI3'kinase-Akt pathway [19]. However the mechanism by which mesenchymal-like tumor cells attenuate their dependence on EGFR signaling and survive as single cells lacking cell-cell junctions has not been actively investigated.

The principle aim of this study was to identify alternative redundant signaling pathways utilized by mesenchymal-like NSCLC lines which might contribute to cell proliferation and survival independently of EGFR. Two models were studied: one where the NSCLC line H358 was induced to undergo an EMT-like transition by exogenous TGFbeta, the other where NSCLC lines with epithelial and mesenchymal phenotypes were compared and contrasted at the molecular level. Mesenchymal NSCLC lines showed attenuation of cell-cell junction and cell-polarity constituents, and increased expression of alternative autocrine signaling which might render EGFR redundant. In H1703 cells autocrine PDGFR and FGFR pathways contributed to activation of PI $3^{\prime} \mathrm{K}-\mathrm{Akt}$ and MekErk pathways, and conversely cell proliferation could be attenuated by PDGFR and FGFR inhibitors. These data implicate EMT as a mechanism for kinase switching, thereby decreasing cellular sensitivity to EGFR inhibition.

\section{Materials and methods}

Cell culture and growth inhibition assay

The human NSCLC lines (H292, H358, H322, H441, A549, Calu6, H460, H1703, SW1573, H266, H522, H650, Calu1 and $\mathrm{H} 23$ ) were cultured in the appropriate ATCC recommended supplemented media. The molecular characteristics of these NSCLC cell lines are detailed at http://www.sanger.ac.uk/ genetics/CGP/cosmic/. For growth inhibition measurements cells were plated and allowed to proliferate for $24 \mathrm{~h}$. Serial dilutions of the indicated drug were added and the cells grown for a further $72 \mathrm{~h}$. Cell viability was determined by chemiluminescent measurement of cellular ATP concentration (CellTiterGlo, Promega; \#G7572). The inhibitors used were erlotinib (Tarceva ${ }^{\mathrm{TM}}$, OSI Pharmaceuticals/Genentech/ Roche), OSI-930 (OSI Pharmaceuticals), PD173074 (SigmaAldrich, \#P2499), CP-673451 (Pfizer).

Immunoblot analysis of NSCLC cell line extracts

Cell lines were treated as indicated in the figure legends. Cell lysates were prepared in RIPA buffer (Sigma, \#R0278) containing protease (Sigma \#P8340) and phosphatase (Sigma, \#P5726) inhibitors. Protein concentration was determined by micro-BCA assay (Pierce, \#23227). Protein immunodetection was performed by electrophoretic transfer of SDS-PAGE separated proteins to nitrocellulose, incubation with antibody and chemiluminescent second step detection (PicoWest; Pierce, \#34078). The antibodies used were : E-cadherin (sc21791), N-cadherin (\#7939), ErbB3 (sc285), pPDGFR (Tyr 754, \#12911), pPDGFR (Tyr720, \#12910), GAPDH (\#25778) and Zeb1 (\#25388), all from Santa Cruz Biotechnology; vimentin (BD550513) and fibronectin (BD610077; both from BD Biosciences); EGFR (\#2232), pEGFR (pTyr1068, \#2234), PDGFR $\alpha$ (\#3164), PDGFR $\beta$ (\#3961), FGFR1 (\#3472), pErk (\#9101), Erk (\#9102), pAkt(ser473, \#9271), Akt (\#9272), all from Cell Signaling Technologies), $\beta$-actin (Sigma, \#A5441).

RNA isolation and RT-PCR analysis

Total RNA was isolated from cell lines (Qiagen, \#74104). First strand cDNA was synthesized and RT-PCR performed (Perkin Elmer, N808-0236) on an Applied Biosystems 7300 real-time PCR instrument.

\section{$\operatorname{TGF} \beta$ treatment of NSCLC cells}

Recombinant TGF $\beta 3$ was added to the cell culture media to a final concentration of $1 \mathrm{nM}$ and was replenished every 23 days. Cell extracts were isolated and analysed as described above. Inhibition of cell proliferation was measured after $48 \mathrm{~h}$ drug treatment by BrdU incorporation and immunodetection (Roche; \#11647229001). Apoptosis induction was monitored after $48 \mathrm{~h}$ drug treatment by caspase 3/7 activation using the CaspaseGlo reagents (Promega, \#G8092).

Preparation of cell extracts and anti-phosphotyrosine affinity capture

Cytosol [F1], membrane [F2] and nuclear [F3]-enriched fractions were prepared using ProteoExtract ${ }^{\circledR}$ reagent ([20]; EMD Bioscience, \#444810). Afterwards, proteins 
were precipitated using trichloroacetic acid/deoxycholate coprecipitation, resuspended in $8 \mathrm{M}$ urea, reduced with $5 \mathrm{mM}$ tributylphosphine (Sigma-Aldrich, \#T7567; $1 \mathrm{~h}$ at RT), alkylated with iodoacetamide $(15 \mathrm{mM}$ for $1.5 \mathrm{~h}$ at $\mathrm{RT}$ ), diluted to $1 \mathrm{M}$ urea and subject to proteolysis with $20 \mu \mathrm{g}$ trypsin (Sigma-Aldrich, \#T6567; 37 ${ }^{\circ} \mathrm{C}, 18 \mathrm{~h}$ ). Peptides were acidified with trifluoroacetic acid (TFA) and desalted using C18 resin. Cell surface capture was performed by biotinylation, solubilization of the membrane lipids and recovery of the crosslinked proteins on streptavidin solid-phase resin (Pierce, \#89881). Samples were adjusted to $8 \mathrm{M}$ urea, reduced, alkylated and proteolytically cleaved with trypsin as described above.

Anti-phosphotyrosine immunoaffinity selection was performed essentially as previously described [21]. Proteins isolated by anti-phosphotyrosine affinity chromatography were reduced, alkylated, cleaved with trypsin and labeled with iTRAQ stable isotope tags as previously described [22, 23] using a different isobaric tag to label peptides from distinct NSCLC cell lines (H292, H358, Calu6, H1703, A549, $\mathrm{H} 460$ and $\mathrm{H} 1650$ ) or different time points following EGFR inhibition with erlotinib. After labeling, the peptides were further fractionated by cation exchange chromatography followed by offline C18 desalting.

Protein identification and quantitation by liquid chromatography-electrospray ionization tandem mass spectrometry

Peptide masses, peptide sequence information and peptide quantitation were obtained by liquid chromatographyelectrospray ionization tandem mass spectrometry (LC-MS/MS) and protein database searching. Informationdependent MS and MS-MS acquisitions were made on a Qq-TOF instrument (Applied Biosystems/MDS Sciex) as previously described [22]. Data were collected using Analyst QS (Version 1.1; Applied Biosystems/MDS Sciex). Proteins were identified at $>95 \%$ confidence from survey and product ion spectra data, searching human sequences within the UniProt protein database (releases from 10/05 to 01/07) using the Paragon algorithm of ProteinPilot (Version 2.0; Builds 44649beta and 50861; Applied Biosystems/MDS Sciex). When multiple isoforms were detected, only peptides specific to each detected form were used, which factored in ion counts for weighting in the protein ratio calculation [24]. Protein identification complied with the guidelines of [25] where 2 or more unique isoform-specific peptides were required for inclusion. Parsimony of protein results was assured by rigorous protein inference with the ProGroup algorithm. Proteins identified with $\geq 95 \%$ confidence with relative abundances between cell states in the upper and lower distribution quartiles $(>75 \%$ or $<25 \%)$ with a $t$-test $P$ value (for any difference between cell line or biological condition) of $<0.05$ were further considered.

\section{Results}

Attenuated EGFR, ErbB2 and ErbB3 signaling in NSCLC lines with a mesenchymal phenotype

Previously we and others have reported that sensitivity of tumor cell lines, carrying wild type EGFR, to EGFR tyrosine kinase inhibitors is correlated with an epithelial to mesenchymal transition of the cells and not with total EGFR abundance or K-Ras mutational status [11-13]. Here, in a large panel of NSCLC lines the mesenchymal phenotype correlated with insensitivity to the selective EGFR inhibitor erlotinib (Fig. 1a), consistent with our previous findings [11]. Implicit in these observations is the suggestion that cells which have undergone an EMT have acquired EGFR-independent mechanisms of cell survival and proliferation. In order to better understand the signaling mechanisms by which mesenchymal-like cells bypass EGFR signaling we compared protein and phosphoprotein expression changes in a panel of NSCLC lines with epithelial and mesenchymal-like phenotypes by quantitative tandem mass spectrometry [22]. Validating this approach, tandem mass spectrometry experiments showed an attenuation of cell polarity and cell junction signaling complexes in cells with a mesenchymal-like cell phenotype (Table 1). We observed reductions in EGFR autophosphorylation at C-terminal sites Y1148 and Y1068 and ErbB2 tyrosine phosphorylation in mesenchymal-like cells compared to epithelial cells.

Next we measured RNA transcript abundance encoding ligands for the EGFR family (TGF $\alpha$, epiregulin, amphiregulin, neuregulin-1, neuregulin-2 and betacellulin). The downregulation of EGFR signaling in the mesenchymal cells correlated with a decrease in autocrine EGF ligand production (Fig. 1b) and decreased EGFR, ErbB2 and ErbB3 phosphorylation and total ErbB3 protein [11, 26]. We asked whether EGFR could be activated by exogenous ligand in mesenchymal like lines. EGF stimulation of EGFR autophosphorylation at Y1068 was observed in all NSCLC lines examined, irrespective of epithelial or mesenchymal phenotype (Fig. 1c). Similarly, EGF stimulation of downstream substrate phosphorylation, for example Erk, Akt (Fig. 1c), Shc and Cbl (data not shown) could be inhibited by addition of the EGFR inhibitor erlotinib in cells with both epithelial and mesenchymal phenotypes. It is important to note that the basal (non-EGF stimulated) levels of phospho-Erk and phospho-Akt could only be inhibited with erlotinib in the epithelial and not the mesenchymal-like cells (Fig. 1c). 


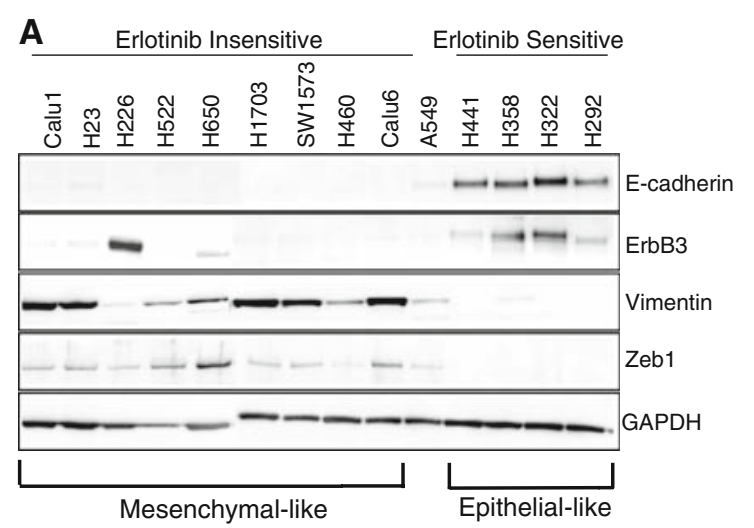

B

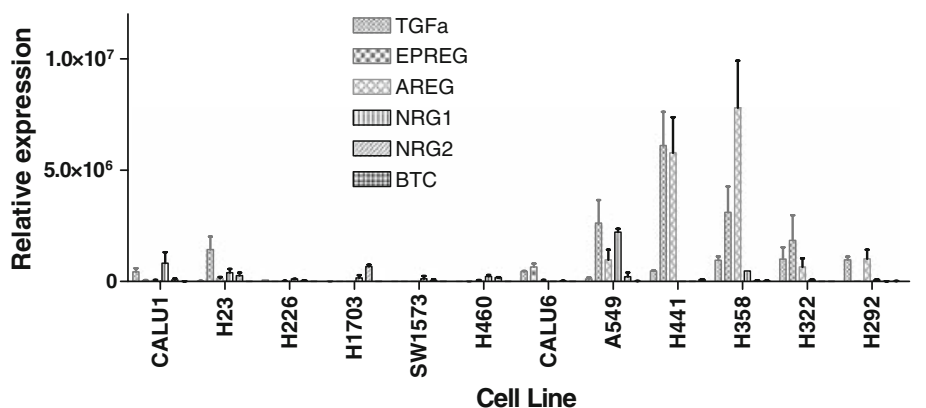

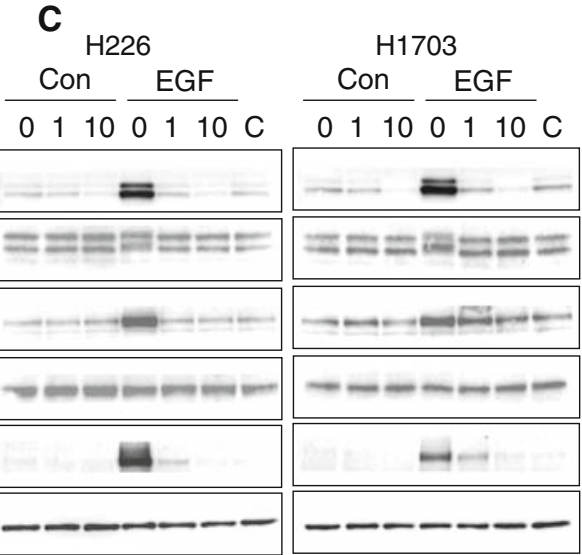

Fig. 1 An EMT in NSCLC cell lines correlates with a reduction in autocrine EGFR signaling. (a) An epithelial marker profile correlates with erlotinib sensitivity in NSCLC cell lines. The indicated cell lines were immunoblotted with antibodies against epithelial markers (Ecadherin and ErbB3) and mesenchymal markers (Zeb1 and vimentin). GAPDH was used as a loading control. (b) Erlotinib insensitive mesenchymal-like cell lines exhibit decreased expression of EGF ligands. Total RNA was prepared form each of the indicated cell lines

\section{Acquisition of PDGFR and FGFR signaling} in mesenchymal NSCLC

We explored the hypothesis that the relative insensitivity of mesenchymal-like NSCLC lines to EGFR TKIs was mediated by the acquisition of alternative proliferative and survival networks. We therefore undertook an analysis of potential pathways that may be bypassing EGFR signaling in these cells. All of the NSCLC lines expressed PDGF ligands, irrespective of whether their phenotype was mesenchymal-like or epithelial. In contrast, none of the four epithelial cell lines expressed either PDGFR $\alpha$ or PDGFR $\beta$, whereas $8 / 13$ of the mesenchymal-like lines expressed one or other of the receptors (Fig. 2a). The expression of the PDGF receptors was further confirmed by immunoblot of NSCLC cell extracts (Fig. 2b). This was of interest as PDGF receptors are normally restricted to cells of mesodermal origin [27]. Interestingly, the mesenchymal NSCLC line H1703 showed markedly increased levels of transcripts and the expression levels of the indicated EGF family ligands were determined by RT-PCR. (c) Basal EGFR signaling is inhibited in epithelial cell lines but not mesenchymal-like cell lines. The indicated cell lines were treated with erlotinib for $2 \mathrm{~h}$ and then left untreated (Con) or stimulated with EGF ( $25 \mathrm{ng} / \mathrm{ml}, 15 \mathrm{~min}$ ). Cell lysates were then probed with the indicated antibodies. Beta-actin was used as a loading control

encoding PDGFR $\alpha$ and PDGF-B ligand (Fig. 2a) relative to the epithelial lines. H1703 cells also exhibited highly elevated tyrosine phosphorylation of PDGFR $\alpha$ and of downstream substrates for example PI3'kinase-p85, STAT3 and SHP-2 (Table 1), whose phosphorylation were reduced in epithelial NSCLC lines. To test active PDGFR coupling to the PI3'kinase pathway, the p85 subunit of PI3'kinase was immunoprecipitated from $\mathrm{H} 1703$ cells and co-precipitating proteins identified by LC-MS/MS. Both $\mathrm{p} 85 \alpha, \mathrm{p} 85 \beta$ and $\mathrm{p} 110 \alpha$ subunits of PI3'kinase were detected and the only receptor tyrosine kinase recovered was PDGFR $\alpha$.

To evaluate the consequences of PDGFR inhibition on the proliferation of mesenchymal-like NSCLC lines, two TKIs were employed to discriminate off target effects. A thiophene $\mathrm{Kit} / \mathrm{Kdr} / \mathrm{Flt} 1 / \mathrm{PDGFR}$ inhibitor (OSI-930) [28] and a chemically distinct, more selective, quinolinyl PDGFR inhibitor (CP673451) [29] were used. From four mesenchymal-like cell lines (H1703, Calu1, Calu6 and H226) significant dose-dependent inhibition of cell growth 
Table 1 Proteins and phosphoproteins differentially expressed between mesenchymal (Calu6, H1703) and epithelial (H292, H358) cell states

\begin{tabular}{|c|c|c|c|c|c|}
\hline \multirow[t]{2}{*}{ Acc } & \multirow[t]{2}{*}{ Protein name } & \multirow{2}{*}{$\begin{array}{l}\text { Isoform-specific } \\
\text { unique peptides } \\
\text { used }\end{array}$} & \multirow{2}{*}{$\begin{array}{l}\text { Sample size } \\
\text { used }\end{array}$} & \multirow[t]{2}{*}{ Cell fraction } & Mesenchymal:Epithelial protein ratios $(\Delta \log 2)$ \\
\hline & & & & & H1703:H292 \\
\hline
\end{tabular}

Receptor tyrosine kinase tyrosine phosphorylation decreased in mesenchymal-like NSCLC cells

$\begin{array}{llrrrrrrr}\text { P00533 } & \text { EGFR pY-1148 } & - & 5 & - & -2.14^{*} & -1.41 & -1.42 \\ & \text { EGFR pY-1086 } & - & 7 & - & -0.72 & -0.95 & -1.05 & -1.28 \\ \text { P04626 } & \text { pY-ErbB2 } & 43 & 185 & \text { pY-B } & -1.01^{* *} & -0.62^{* *} & -1.49^{* *} & -1.11^{* *} \\ & & 20 & 83 & \text { pY-S } & -0.43^{* *} & -0.33^{*} & -1.38^{* *} & -1.32^{* *} \\ \text { P07949 } & \text { pY-Ret } & 10 & 13 & \text { pY-B } & 2.24^{*} & \text { nd } & 1.26 & \text { nd } \\ & & 9 & 27 & \text { pY-S } & 1.65^{* *} & \text { nd } & 2.07 * * & \text { nd }\end{array}$

PDGFR auto and substrate phosphorylation increased in mesenchymal-like H1703 cells

$\begin{array}{llrrlll}\text { P16234 } & \text { pY-PDGF-R } \alpha & 13 & 22 & \text { pY-B } & 1.44^{* *} & 1.58^{* *} \\ \text { P42338 } & \text { pY-P13K p100 } \beta & 6 & 8 & \text { pY-B } & 1.17 & 1.52^{* *} \\ \text { P27986 } & \text { pY-P13K p85 } \alpha & 7 & 22 & \text { pY-B } & 1.57 & 1.90 \\ \text { Q06124 } & \text { pY-SHP2 } & 24 & 61 & \text { pY-B } & 0.83^{* *} & 1.20^{* *} \\ \text { P40763 } & \text { STAT3 } & 1 & 5 & \text { F2 } & 1.08^{* *} & 1.82^{* *} \\ & & 4 & 4 & \text { pY-B } & 1.85 & 1.57 \\ & & 4 & 18 & \text { F1 } & 1.19 * * & 1.84 * *\end{array}$

Representative lineage-specific markers altered in mesenchymal-like NSCLC cells

\begin{tabular}{|c|c|c|c|c|c|c|c|c|}
\hline \multirow[t]{3}{*}{ P31947 } & 14-3-3s (Stratifin) & 12 & 86 & $\mathrm{~F} 1$ & $-2.26 * *$ & $-2.69 * *$ & $-1.87 * *$ & $-2.25^{* *}$ \\
\hline & & 4 & 19 & $\mathrm{~F} 2$ & $-1.63 * *$ & $-2.24 * *$ & $-1.16^{* *}$ & $-1.74 * *$ \\
\hline & & 5 & 6 & pY-B & -0.40 & -2.37 & -0.35 & -1.04 \\
\hline \multirow[t]{2}{*}{ P12830 } & E-Cadherin & 1 & 1 & $\mathrm{~F} 2$ & -2.30 & -1.99 & -2.44 & -2.04 \\
\hline & & 20 & 64 & pY-B & $-1.35 * *$ & $-1.44 * *$ & $-0.99 * *$ & $-0.94 * *$ \\
\hline P22223 & P-Cadherin & 4 & 6 & pY-B & $-2.14^{*}$ & -1.63 & $-1.45^{*}$ & -0.97 \\
\hline \multirow[t]{3}{*}{ P35221 } & $\alpha$-Catenin & 10 & 60 & $\mathrm{~F} 2$ & $-1.50 * *$ & $-1.42 * *$ & $-1.39 * *$ & $-1.58 * *$ \\
\hline & & 94 & 518 & pY-B & $-1.56^{* *}$ & $-1.68 * *$ & $-1.03 * *$ & $-1.23 * *$ \\
\hline & & 22 & 66 & pY-S & $-0.85^{* *}$ & $-0.77 * *$ & $-0.90 * *$ & $-0.87 * *$ \\
\hline \multirow[t]{3}{*}{ P35222 } & $\beta$-Catenin & 10 & 41 & $\mathrm{~F} 2$ & $-0.91 * *$ & $-1.89 * *$ & $-0.90 * *$ & $-1.94 * *$ \\
\hline & & 25 & 92 & pY-B & $-1.22 * *$ & $-0.98 * *$ & $-0.67 *$ & -0.43 \\
\hline & & 9 & 15 & pY-S & $-0.59 * *$ & $-0.65 * *$ & $-0.51^{*}$ & $-0.63 * *$ \\
\hline \multirow[t]{3}{*}{ O60716 } & $\delta$-Catenin & 9 & 27 & $\mathrm{~F} 2$ & $-0.76^{* *}$ & $-0.84 * *$ & $-0.74 * *$ & $-0.84 * *$ \\
\hline & & 105 & 765 & pY-B & $-1.50 * *$ & $-1.81 * *$ & $-0.90 * *$ & -1.24 \\
\hline & & 28 & 191 & pY-S & $-0.99 * *$ & $-1.25 * *$ & $-1.47 * *$ & $-1.69 * *$ \\
\hline \multirow[t]{3}{*}{ P08670 } & Vimentin & 18 & 75 & $\mathrm{~F} 2$ & $0.94 *$ & $1.12 * *$ & $1.50 * *$ & $1.56^{* *}$ \\
\hline & & 103 & 908 & pY-B & $2.74 * *$ & $2.59 * *$ & $2.77 * *$ & $2.66^{* *}$ \\
\hline & & 41 & 466 & pY-S & $2.08 * *$ & $2.10 * *$ & $2.30 * *$ & $2.25 * *$ \\
\hline
\end{tabular}

Proteins significantly decreased in either mesenchymal-like lines Calu6 or H1703, are expressed as log2 ratios relative to both epithelial cell backgrounds (denominators H292 and H358). At least two biological experiments were performed (N1 and N2) each of which was analyzed in multiple ion exchange fractions in 2-3 LC-MS/MS experiments per fractions, expressed as log2. Proteins and phosphoproteins identified from two biological experiments with a $95 \%$ confidence interval were quantitated and mesenchymal to epithelial ratios determined $(\Delta \log 2)$ together with its statistical significance $(*<0.05 ; * *<0.01)$. The number of isoform-specific unique peptides and total peptides used for quantitation (sample size) are indicated. Cell fractions included cytoplasmic-enriched (soluble; F1) and membrane-enriched (particulate; F2). Phosphoprotein fractions included steady-state (pY-B) or serum-stimulated (pY-S) conditions. nd; not detected (i.e. cell specific)

was only observed in H1703 cells (Fig. 3a). OSI-930 inhibited both basal and PDGF-stimulated PDGFR $\alpha$, Erk and Akt activation in $\mathrm{H} 1703$ cells but not in the epithelial control line H292 lacking PDGFR (Fig. 3b). H1703 response to PDGFR inhibition may derive from relatively high expression of PDGF-BB ligand and the creation of a strong autocrine signaling loop. $\mathrm{H} 226$ and Calu1, shown to express PDGFR $\beta$ but little PDGF-B chain, did not show autocrine PDGFR activation and were not growth inhibited by either of the two distinct PDGFR inhibitors. 


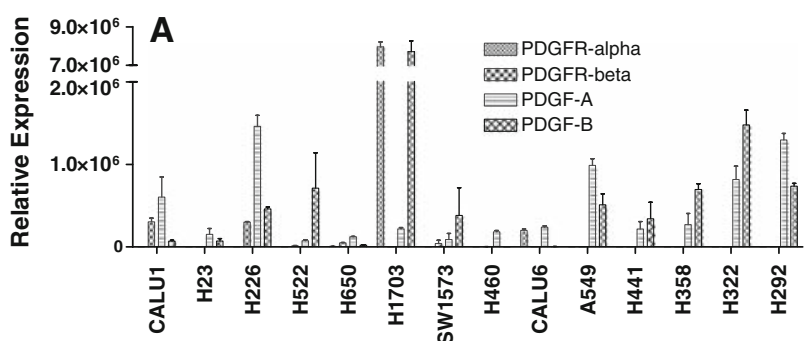

B

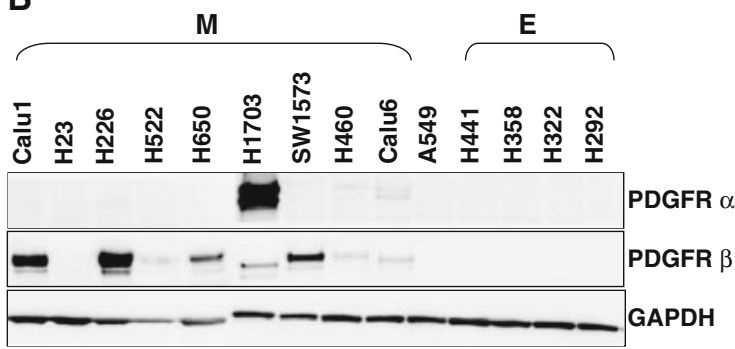

Fig. 2 Acquisition of PDGFR signaling in mesenchymal-like NSCLC lines. (a) NSCLC cell lines express PDGF receptors and their cognate ligands. Total RNA was prepared from the indicated cell lines and the expression level of PDGF receptors and PDGFs were measured by RT-PCR. (b) Expression of PDGF receptors in mesenchymal-like NSCLC cell lines. Protein extracts from the indicated cell lines were analysed by immunoblot with the indicated antibodies

We next examined whether interaction between PDGFR and EGFR signaling pathways could be observed. The H1703 line expressed relatively high levels of both PDGFR and EGFR, and showed increased PDGFR autophosphorylation and substrate-phosphorylation when EGFR was inhibited by erlotinib. EGFR blockade significantly increased phosphorylation of multiple PDGFR substrates, notably PDGFR $\alpha$ itself, PI3'kinase p85 and p110 subunits, SHP-2, COOL-2 and PLC $\gamma$ (Fig. 3c). The EGFR TKI mediated increase in PDGFR autophosphorylation was confirmed by immunoblot using anti-phosphoPDGFR antibodies to $\mathrm{Y}^{754}$ and $\mathrm{Y}^{720}$ (Fig. 3d).

We determined the relative abundance of RNA transcripts encoding receptor tyrosine kinases and signaling pathways which might activate Akt and/or Erk in an EGFR-independent manner. RT-PCR measurements of FGFR levels indicated FGFR1 was the predominant isoform expressed in mesenchymal-like NSCLC cell lines (Fig. 4a). Immunoblot of NSCLC line lysates with antiFGFR antibodies confirmed FGFR1 was expressed predominately in the mesenchymal-like cell lines (data not shown). Three mesenchymal-like NSCLC cell lines H1703, Calu1 and H226, showing elevated expression of FGFR1, 3 and 4 were treated with increasing concentrations of a specific inhibitor of FGFR1 and FGFR3 (PD173074, Sigma) and cell proliferation measured (Fig. 4b). H1703 and H226 cells were growth inhibited by treatment with
PD173074, with little effect observed in Calu1 cells. FGFR inhibition had an effect primarily upon Erk signaling and to a lesser extent on Akt signaling in H1703 and H226 cells whereas no effect was observed in the epithelial control line H292 (Fig. 4c). H226 mesenchymal-like cells showed increased FGFR abundance and were growth inhibited by FGFR blockade, while H1703 utilized both FGFR and PDGFR pathways to activate the PI3'kinase and Ras pathways and stimulate cell proliferation.

Cell signaling changes in $\mathrm{H} 358$ cells undergoing an EMT-like transition

We next asked whether the changes in signaling axes used in mesenchymal-like cells compared to epithelial cells could be initiated by an EMT-like transition. To this end we developed an isogenic in vitro EMT model wherein activation of the TGF $\beta$ pathway induced a fibroblastic transition in vitro $[2,30]$. Exposure of $\mathrm{H} 358$ cells to TGF $\beta$ $(1 \mathrm{nM})$ over 14 days resulted in a marked reduction in Ecadherin and ErbB3, coupled to an increased expression of the mesenchymal markers vimentin and Zeb1 (Fig. 5a), a scattered cellular phenotype and increased motility (data not shown). These phenotypic changes could be prevented by co-treatment with the $\mathrm{TGF} \beta$ receptor inhibitor SB431542 (data not shown) and reversed by removal of $\operatorname{TGF} \beta$ from the culture media (Fig. 5a). Consistent with reports that TGF $\beta$-inducible EMT in vitro is a rare event [30], a complete transition was observed only in H358 cells. A partial EMT phenotype could be achieved in H322 and A549 cells after 14 days (data not shown).

We next examined the effects of EMT on cellular sensitivity to EGFR inhibition. H358/TGF $\beta$ cells were less sensitive to the growth inhibitory effects of erlotinib than parental $\mathrm{H} 358$ cells, showing an approximate 7 fold shift in half-maximal effective concentration (Fig. 5b). In addition, H358/TGF $\beta$ cells were approximately 1.5 fold less sensitive to erlotinib-induced apoptosis (Fig. 5b). EGFR TKI treatment of $\mathrm{H} 358$ cells resulted in inhibition of the basal phosphorylation of Erk (Fig. 5c). In contrast H358/TGF $\beta$ cells exhibited elevated steady-state activation of Erk (as measured by phosphorylation $\mathrm{T}^{202} / \mathrm{Y}^{204}$ ) which could only partially be inhibited by EGFR blockade (Fig. 5c). In control experiments where TGF $\beta$-induced EMT was blocked by co-treatment with the TGF $\beta$ receptor inhibitor SB431542, Erk phosphorylation was inhibited by EGFR blockade in a similar fashion to the parental control H358 cells (data not shown).

To determine whether the EMT induced changes in the H358/TGFbeta model were reflective of those observed in established mesenchymal-like NSCLC we measured the expression of EMT marker genes by RT-PCR (Fig. 6a). We observed an increase in the expression of transcription 

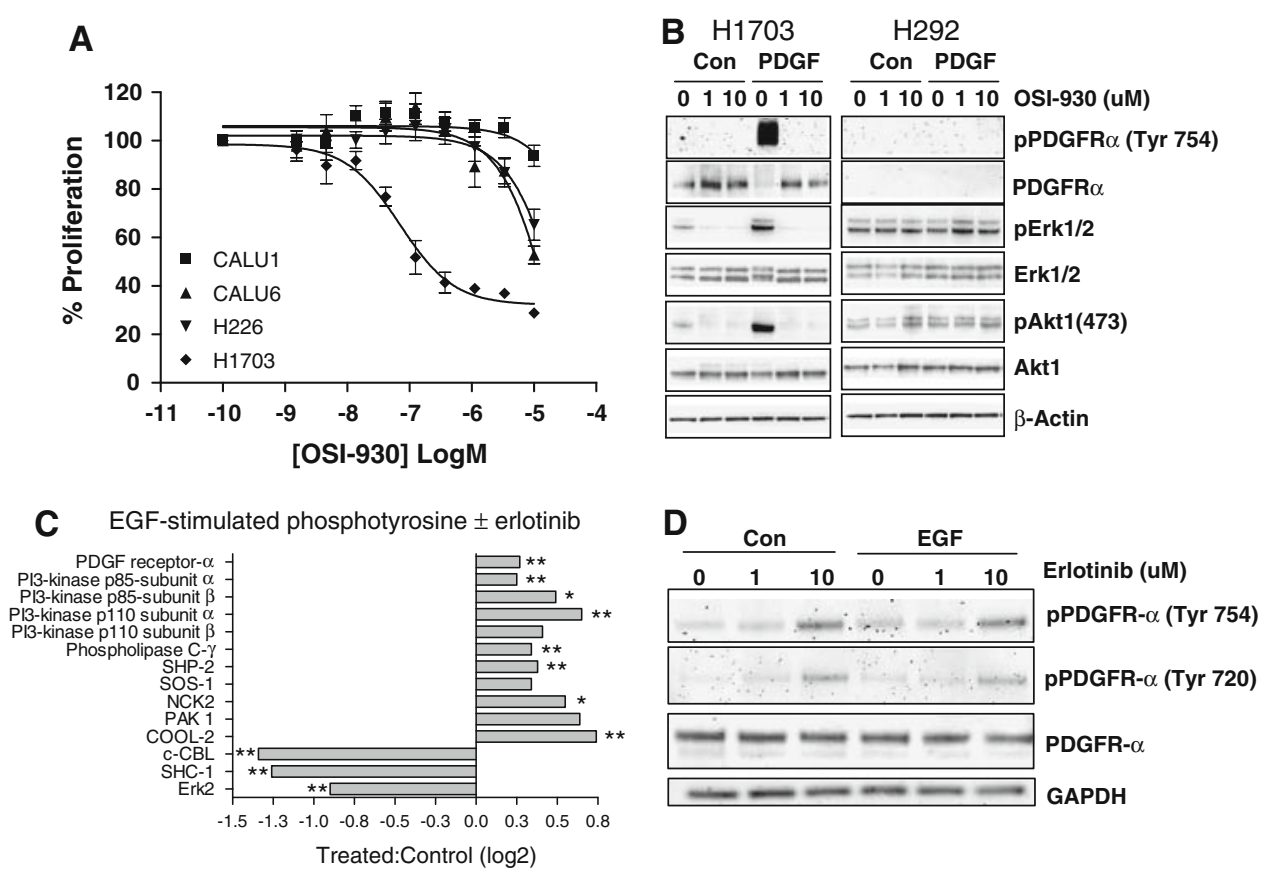

Fig. 3 Autocrine PDGFR signaling regulates cell proliferation in mesenchymal-like NSCLC line H1703. (a) Inhibition of PDGFR signaling in $\mathrm{H} 1703$ cells inhibits cell proliferation. Cells were plated in 96 well dishes and treated with increasing concentrations of OSI930. Cell proliferation was measured after $72 \mathrm{~h}$. Data from 3 independent experiments (mean $\pm \mathrm{SEM}$ ) is plotted as percentage of the untreated control. (b) Inhibition of PDGFR $\alpha$, Erk and Akt signaling by a PDGFR antagonist in the mesenchymal-like NSCLC cell line $\mathrm{H} 1703$ vs. the epithelial control line $\mathrm{H} 292$. The indicated cell lines were treated with drug (OSI-930, 0,1 or $10 \mu \mathrm{M}, 2 \mathrm{~h}$ ) and then left untreated or treated with PDGF (10 ng/ml, $10 \mathrm{~min})$. Cell lysates were prepared and protein extracts analysed by western blot with the

factors implicated in the EMT process, such as Snail, Zeb1 and Zeb2 [31]. H358/TGF $\beta$ cells expressed reduced levels of EGF family ligands, in particular TGF $\alpha$, epiregulin and amphiregulin (Fig. 6a), consistent with the observed down regulation of EGF ligands in the committed mesenchymallike NSCLC cells (Fig. 1b). In addition, again consistent with the committed mesenchymal-like cells, we observed an up regulation of the RNA transcripts encoding PDGFR $\alpha$, PDGFR $\beta$, PDGF-A and FGFR1 in the H358/ TGF $\beta$ cells relative to their parental counterpart (Fig. 6a). To determine whether these receptors were functional in the transitioned cells we stimulated H358 cells, which had been induced to undergo EMT (Fig. 6b), with either PDGF-AA/BB or acidic FGF/basic FGF ligand mixtures. Extracts were immunoprecipitated with an anti-phosphotyrosine antibody and then probed for the presence of either PDGFR or FGFR1 (Fig. 6c). Both PDGFR and FGFR1 were shown to be activated and functional in response to ligand only in cells in which an EMT-like transition was observed.

indicated antibodies. (c) Inhibition of EGFR signaling in H1703 cells increases PDGFR signaling. H1703 cells were either untreated or treated with erlotinib $(10 \mu \mathrm{M}, 2 \mathrm{~h})$ then stimulated with EGF (10 ng/ $\mathrm{ml}, 10 \mathrm{~min}$ ) and phospho-proteins captured using an anti-phosphotyrosine antibody. The recovered proteins were analysed by LC-MS/ MS and changes in PDGFR signaling associated proteins expressed as $\log 2$ changes between treated and control samples $(* P<0.05$; ** $P<0.01$ ). (d) $\mathrm{H} 1703$ cells were treated with the indicated concentrations of erlotinib $(2 \mathrm{~h})$ and then left untreated (Con) or stimulated with EGF (10 ng/ml, $10 \mathrm{~min})$. Cell extracts were analysed by western blotting with the indicated antibodies

\section{Discussion}

Tumor-derived cells, likely located at the tumor-stromal interface, can transition to a more migratory invasive state through activation of specific biological pathways resembling an epithelial-to-mesenchymal-like transition [6]. This transition can allow the cells to escape the site of primary tumor formation, resist anokis and seed in distant sites prior to development of a metastatic lesion. The similarity of stroma and adjacent tumor has been reported in colon carcinoma [32]. This phenotypic behavior is associated with extensive genetic reprogramming, most notable through the actions of a family of transcription factors such as Snail, Zeb [31], FOXC2, HMGA and Twist. These factors play critical roles in mesoderm formation during embryonic development and can protect cells from apoptosis in vitro $[33,34]$. The sensitivity of tumor cells to the EGFR tyrosine kinase inhibitor erlotinib has been reported to correlate with EMT status in multiple cancer lines. These observations provided protein markers that were 


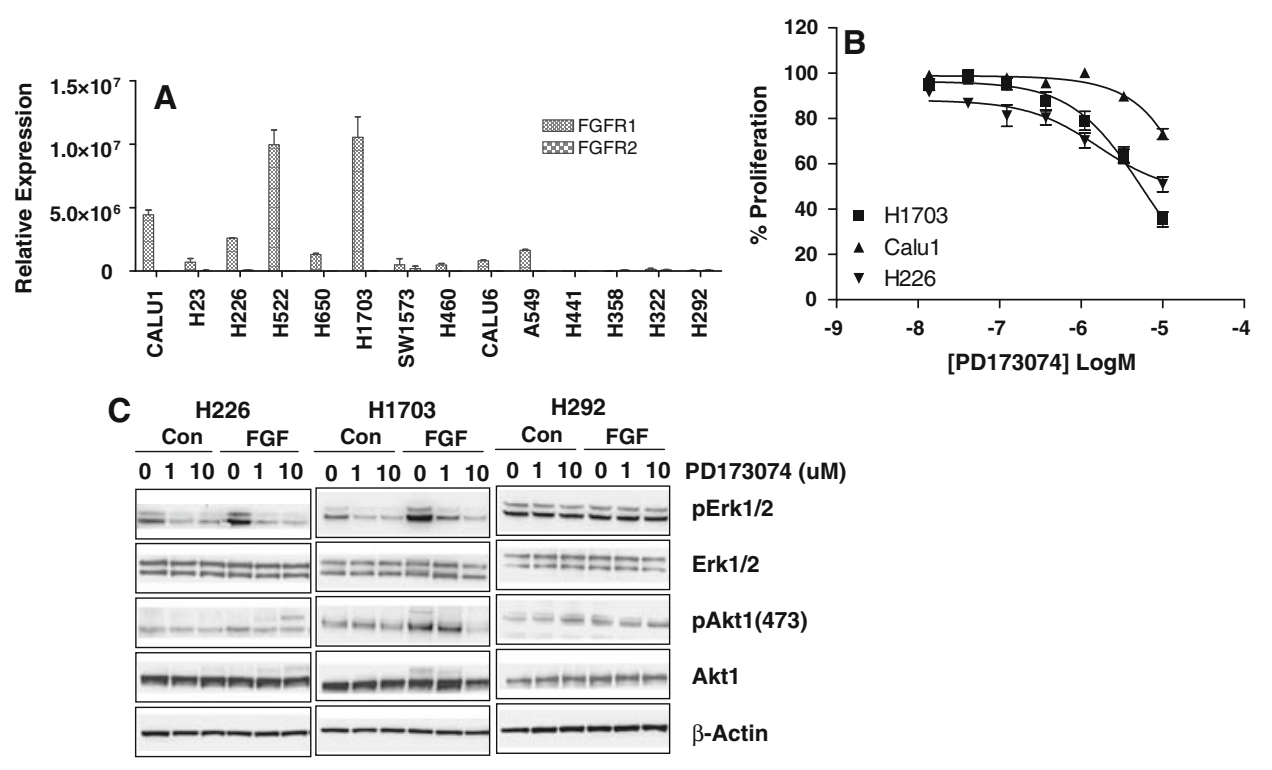

Fig. 4 Acquisition of FGF signaling in mesenchymal-like NSCLC cell lines.; (a) NSCLC cell lines express FGF receptor-1. Total RNA was prepared from the indicated cell lines and the expression level of FGF receptors were determined by RT-PCR. (b) Inhibition of FGF signaling in mesenchymal-like NSCLC cell lines inhibits cell proliferation. Cells were plated in 96 well dishes and treated with increasing concentrations of PD173074. Cell proliferation was measured after $72 \mathrm{~h}$. Data from 3 independent experiments

able to predict sensitivity to the actions of the drug, but did not provide a mechanistic explanation as to the reason why mesenchymal-like cells were less sensitive.

We have undertaken a molecular analysis of the mesenchymal-like and epithelial NSCLC cell lines to establish a mechanistic basis for the differential sensitivity. Phospho-proteomic measurements of the cell lines gave an indication that EGFR signaling was attenuated in the mesenchymal-like lines, through the decreased levels of pEGFR and pErbB2 peptides recovered, and this was linked to the decreased levels of EGF ligand expression in these cell lines. This observation, coupled with the decrease in ErbB3 levels in the mesenchymal lines, gives a strong rationale for the observed decreased erlotinib sensitivity. These data suggested a significant down-regulation of the EGFR, ErbB2 and ErbB3 signaling network in cells which have undergone an EMT compared to their epithelial counterparts. Importantly we have also demonstrated that this shift in flux through the EGFR pathway is a direct result of the EMT, as treatment of $\mathrm{H} 358$ cells with TGF $\beta$ results in all the hallmarks of EMT, including reduced EGFR inhibitor sensitivity, and a down regulation of EGF ligand production and ErbB3 protein levels. Together these data suggest that epithelial tumor cells have a strong dependency upon autocrine EGF signaling for cell survival and proliferation and that as they undergo an EMT this dependency is lost due to a down regulation of autocrine (mean \pm SEM) was plotted as a percentage of the untreated control. (c) Inhibition of Erk and Akt signaling by an FGFR antagonist in mesenchymal-like NSCLC cell lines. The indicated cell lines were treated with drug (PD173074; 0, 1 or $10 \mu \mathrm{M}, 2 \mathrm{~h}$ ) and then left untreated or treated with basic FGF (50 ng/ml, $10 \mathrm{~min}$ ). Cell lysates were prepared and protein extracts analysed by western blot with the indicated antibodies

EGF ligand production and a down regulation of ErbB3 protein levels. Paradoxically chronic activation of EGFR signaling can promote EMT-like transitions [35, 36], but once having occurred, these pathways are no longer activated and mesenchymal tumor cells acquire alternative survival signals.

These data led us to set the hypothesis that the mesenchymal-like erlotinib insensitive cell lines had acquired alternative modes of signaling for survival and proliferation. In NSCLC lines we correlated EMT to the acquisition of autocrine PDGFR and FGFR signaling, further defining the mechanism for insensitivity of mesenchymal-like NSCLC cell lines to EGFR blockade. All these cell lines were derived from EGFR TKI-therapy naive patients and so have acquired these alternative signaling mechanisms as a consequence of tumor progression and EMT. The aberrant expression of PDGFRs in epithelial-derived carcinoma cells is less well studied. In stable mesenchymal NSCLC lines with autocrine PDGFR or FGFR activation (eg. H1703 and H226), onco-addiction to these receptor tyrosine kinases could be observed. We show a strong dependence on PDGFR signaling in $\mathrm{H} 1703$ cells, where PDGFR inhibition led to decreased activation of PI3K-Akt and Mek-Erk pathways and reduced proliferation. Recently Rikova et al. [37] reported similar observations to that presented here and in addition showed that $\mathrm{H} 1703$ xenografts were sensitive to the Abl/Kit/PDGFR inhibitor 

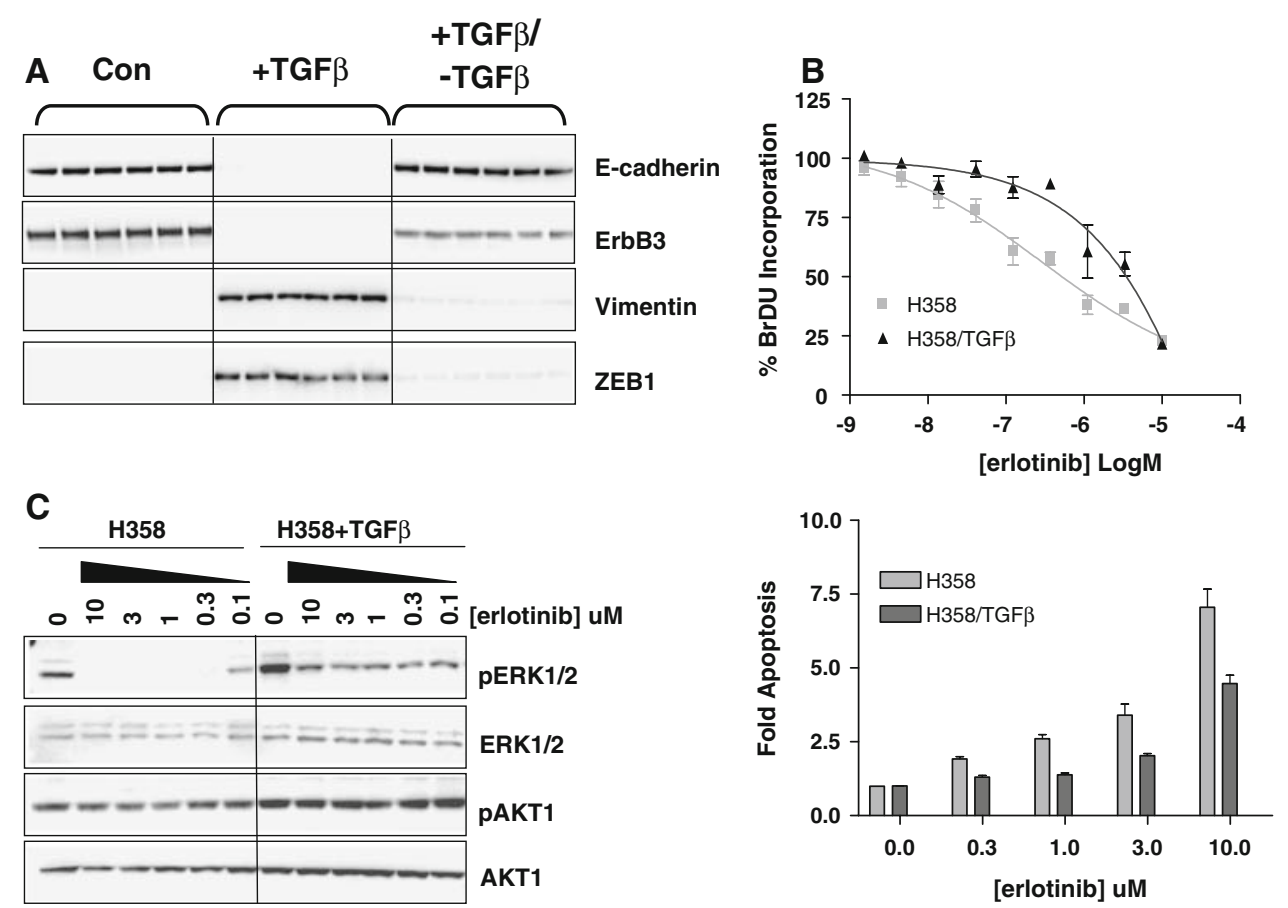

Fig. 5 TGF $\beta$ induces EMT in H358 cells and reduces erlotinib sensitivity. (a) TGF $\beta$-induces a reversible EMT in H358 cells. H358 cells (Con) were treated with $\operatorname{TGF} \beta 3(+\mathrm{TGF} \beta)$ and then TGF $\beta 3$ removed from the culture media $(+\mathrm{TGF} \beta /-\mathrm{TGF} \beta)$. Protein extracts were analysed by western blotting with the indicated antibodies. A representation of the transition from epithelial to mesenchymal and back to epithelial phenotypes is shown below. (b) TGF $\beta$-induced EMT results in reduced sensitivity to erlotinib-mediated growth inhibition and apoptosis. H358 cells were treated with TGF $\beta(1 \mathrm{nM}$, 21 days) plated in 96 well dishes and treated with increasing concentrations of erlotinib. Cell proliferation was measured $48 \mathrm{~h}$

imatinib, further supporting a role for PDGFR in this NSCLC cell line. The majority of the mesenchymal-like NSCLC lines expressed PDGFR $\beta$, however these lines did not express PDGF ligands, and so PDGFR $\beta$ was not autophosphorylated and cells were not inhibited by PDGFR receptor inhibitors. However functional PDGF receptor on these mesenchymal-like cell lines could potentially be activated by paracrine PDGF within an in vivo tumor microenvironment, not mimicked in vitro. Of note is that the metastable cells induced to undergo EMT by TGF $\beta$ treatment did not show a dependency upon autocrine PDGFR signaling (data not shown), but were clearly able to respond to ligand stimulation, suggesting a potential paracrine role for PDGFR signaling in the mesenchymallike state in vivo. The expression of PDGFRs in mesenchymal-like NSCLC lines is consistent with increased PDGF signaling in mouse mammary cells induced to undergo EMT [38], where it was observed to be of critical importance in maintaining the mesenchymal-like state. Further, expression of both forms of PDGFR has been reported in late stage human mammary carcinomas [39] after erlotinib treatment by analyzing BrdU incorporation and data from 2 independent experiments is plotted as a percentage of the untreated control. Apoptosis was measured 48 hours after erlotinib treatment by caspase 3/7 activation. Data from 2 independent experiments is plotted as fold induction over the untreated control. (c) TGF $\beta$-induced EMT results in increased basal Erk1/2 activity and reduced erlotinib inhibition of Erk1/2 kinase activity. H358 cells were treated with $\operatorname{TGF} \beta$ as in (a). The cells were then treated with increasing concentrations of erlotinib for $2 \mathrm{~h}$. Protein extracts were analysed by western blot with the indicated antibodies

implying a role in more advanced aggressive disease, a scenario in which EMT is particularly relevant [6].

The acquisition of FGFR expression is equally interesting, as again this receptor has primarily been studied in the context of its role in angiogenesis. The presence of FGFR1 in mesenchymal-like cells coupled to its increased gene expression during TGF $\beta$-induced EMT strongly implicates it in a role in mesenchymal-like cell function, and here we have shown two NSCLC cell lines (H1703 and H226) which utilize autocrine FGFR signaling for proliferation. In addition cells that are induced to undergo an EMT exhibit acquisition of functional FGFR1 suggesting a potential role for paracrine FGFR signaling in mesenchymal-like cells. FGF2 has been reported to stimulate an EMT in vitro [40] and tumor cells exhibiting autocrine FGFR signaling were mesenchymal in nature [41] consistent with the results presented here. Similarly an interaction between FGFR1 and the mesenchymal marker N-cadherin has been reported to be important in regulating ERK signaling, MMP9 expression and the invasive phenotype of breast cancer cells [42]. 

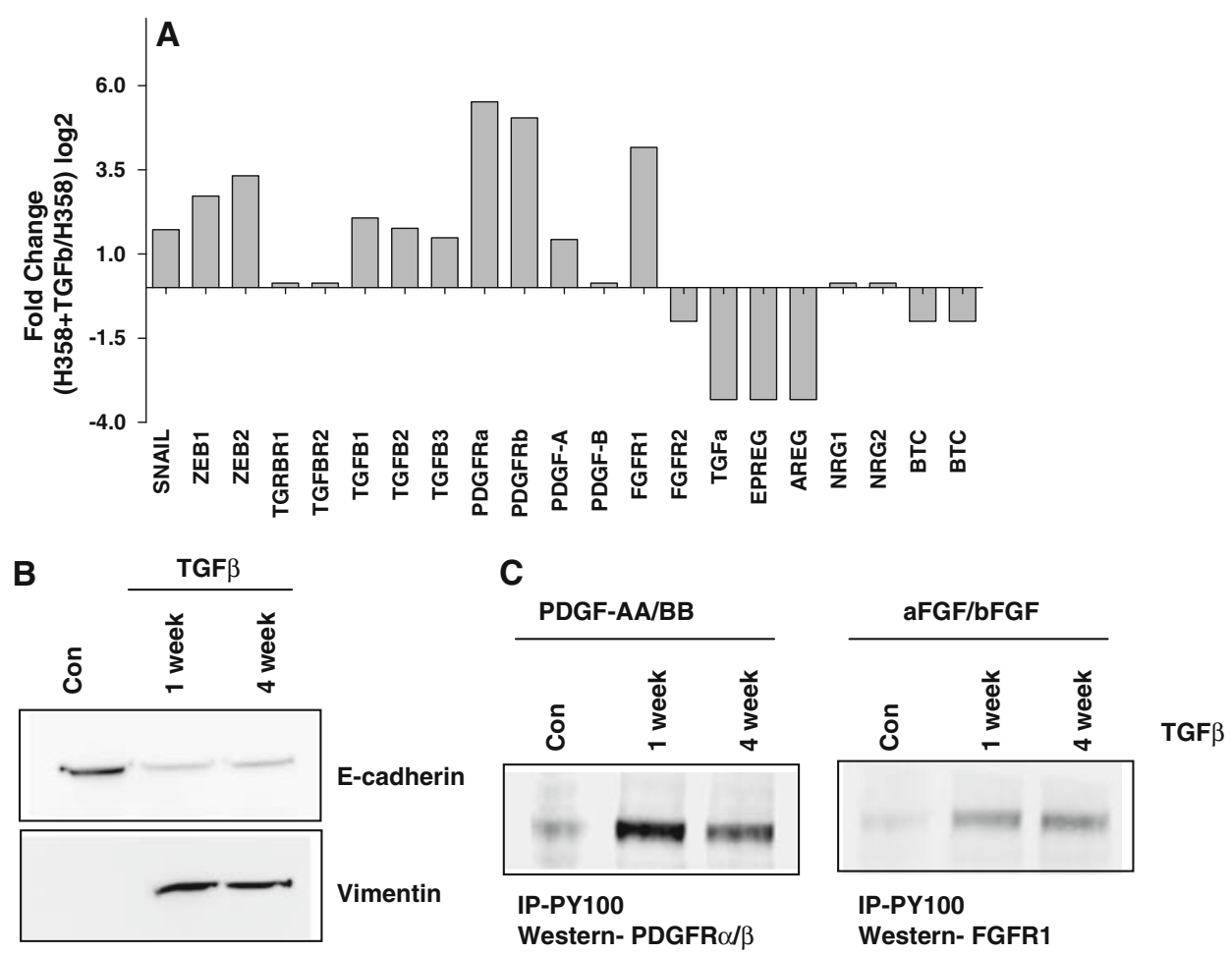

Fig. 6 Metastable mesenchymal-like H358/TGF $\beta$ cells initiate functional PDGFR and FGFR expression. (a) Gene expression changes upon TGF $\beta$-induced EMT in H358 cells. H358 cells were treated with TGF $\beta$ for 7 days and total RNA was isolated from the cells. Expression of the indicated genes was analysed by RT-PCR and the fold change in expression in TGF $\beta$-treated cells compared to control cells determined. Data from 2 independent experiments is plotted as $\log 2$ fold change on the $Y$ axis, with positive values indicating an increase and negative values indicating a decrease in gene expression in the TGF $\beta$-treated cells. (b) H358 cells were treated with TGF $\beta$

Crosstalk between EGFR and PDGFR pathways was observed in the mesenchymal cell line H1703. Inhibition of EGF stimulated EGFR signaling by erlotinib in H1703 cells induced phosphorylation and activation of PDGFR and downstream signaling. Cross-talk between EGFR and Met RTKs [43] and between EGFR and IFG1R RTKs [44] has been previously reported. This further reinforces the notion of EGFR TKI therapy being of limited effectiveness against that fraction of tumor cells which have undergone an EMT-like transition. Whether the increased PDGFR signaling relates to EGFR-mediated saturation of internalization, as has been suggested in v-src transformation [45], remains to be determined.

Our observations contrasting $\mathrm{H} 358$ and $\mathrm{H} 358 / \mathrm{TGF} \beta$ isogenic lines as a model of metastable EMT-like transition in vitro further recapitulated many of the findings with established stable NSCLC lines. However, one important difference was the reversibility of the process, in this case by removal of TGF $\beta$ from the culture medium. Although the TGF $\beta$-induced EMT can be blocked by inhibition of $\operatorname{TGF} \beta$ signaling with a small molecule inhibitor, the
$(1 \mathrm{nM})$ for 1 week or 4 weeks and protein extracts analyzed for the expression of E-cadherin and vimentin. (c) TGF $\beta$-induced EMT of $\mathrm{H} 358$ cells induces the expression of functional PDGFR and FGFR receptors. H358 cells treated with TGF $\beta$ for 1 or 4 weeks were treated with PDGF-AA/BB (10 ng/ml, $10 \mathrm{~min})$ or acidic FGF/basic FGF $(10 \mathrm{ng} / \mathrm{ml}, 10 \mathrm{~min})$ and extracts immunoprecipitated with an antiphosphotyrosine antibody (PY-100). Immunoprecipitated proteins were analyzed for the presence of PDGFR $\alpha / \beta$ or FGFR1 by immunoblot

established mesenchymal-like NSCLC cell lines could not be induced to revert to an epithelial cell phenotype even upon prolonged TGF $\beta$ receptor inhibition (data not shown). Interestingly initial data also suggest that when $\operatorname{TGF} \beta$ induced mesenchymal-like cells revert back to an epithelial state, EGFR activation and EGFR TKI sensitivity are not immediately restored suggesting some memory of signaling changes associated with the EMT transition persist in the absence of mesenchymal markers (data not shown). It was been proposed that upon arrival at a secondary site, mesenchymal cells can revert to an epithelial state and reinitiate a proliferative signaling program [6]. The TGF $\beta$ induced EMT model may therefore be a better reflection of the metastable EMT process, whereby continued exogenous $\operatorname{TGF} \beta$, or by analogy paracrine signaling, is required to maintain the mesenchymal-like state. These data also suggest that the established mesenchymal-like NSCLC lines have become epigenetically arrested in the mesenchymal state, potentially analogous to spindle cell carcinomas of the lung which reflect $\sim 2 \%$ of NSCLC cases. 
We suggest a model where tumor cells can utilize multiple mechanisms to bypass EGFR dependence. In an epithelial state EGFR TKI resistance mechanisms include IGF1R activation, Met amplification and EGFR T790M mutation. In cells with a more mesenchymal state EGFR TKI resistance can be correlated with autocrine PDGFR and FGFR signaling. Recent data suggest patients with metastasis can have heterogeneous tumors that can contain epithelioid cells within the tumor and mesenchymal-like derived from an EMT-like transition cells at the tumorstroma interface [46]. Specific targeted therapies directed against mesenchymal-like cell survival pathways would be predicted to reduce tumor metastasis and progression. Therefore, the observation that tumors can acquire PDGFR and FGFR autocrine signals through an EMT-like transition, suggest new therapeutic modalities to target both epithelial and mesenchymal tumor phenotypes that may support cancer progression and recurrence [47].

Acknowledgements We thank the OSI-930 project team, Neil Gibson, Liz Buck, Sharon Barr, Ken Iwata, and Mark Miglarese for critical discussion. We thank Michael J Comb and Klarisa Rikova (Cell Signaling Technologies) for open sharing of data and discussion prior to publication.

Open Access This article is distributed under the terms of the Creative Commons Attribution Noncommercial License which permits any noncommercial use, distribution, and reproduction in any medium, provided the original author(s) and source are credited.

\section{References}

1. Gupta GP, Massague J (2006) Cancer metastasis: building a framework. Cell 127(4):679-695. doi:10.1016/j.cell.2006.11.001

2. Oft M, Peli J, Rudaz C et al (1996) TGF-betal and Ha-Ras collaborate in modulating the phenotypic plasticity and invasiveness of epithelial tumor cells. Genes Dev 10(19):2462-2477. doi:10.1101/gad.10.19.2462

3. Perl AK, Wilgenbus P, Dahl U et al (1998) A causal role for E-cadherin in the transition from adenoma to carcinoma. Nature 392(6672):190-193. doi:10.1038/32433

4. Brabletz T, Jung A, Spaderna S et al (2005) Opinion: migrating cancer stem cells - an integrated concept of malignant tumour progression. Nat Rev Cancer 5(9):744-749. doi:10.1038/nrc1694

5. Christofori G (2006) New signals from the invasive front. Nature 441(7092):444-450. doi:10.1038/nature04872

6. Thiery JP (2002) Epithelial-mesenchymal transitions in tumour progression. Nat Rev Cancer 2(6):442-454. doi:10.1038/nrc822

7. Matsumura T, Makino R, Mitamura K (2001) Frequent downregulation of E-cadherin by genetic and epigenetic changes in the malignant progression of hepatocellular carcinomas. Clin Cancer Res 7(3):594-599

8. Yoshiura K, Kanai Y, Ochiai A et al (1995) Silencing of the E-cadherin invasion-suppressor gene by $\mathrm{CpG}$ methylation in human carcinomas. Proc Natl Acad Sci USA 92(16):7416-7419. doi:10.1073/pnas.92.16.7416

9. Baumgart E, Cohen MS, Neto BS et al (2007) Identification and prognostic significance of an epithelial-mesenchymal transition expression profile in human bladder tumors. Clin Cancer Res 13(6):1685-1694. doi:10.1158/1078-0432.CCR-06-2330

10. Willipinski-Stapelfeldt B, Riethdorf S, Assmann V et al (2005) Changes in cytoskeletal protein composition indicative of an epithelial-mesenchymal transition in human micrometastatic and primary breast carcinoma cells. Clin Cancer Res 11(22):80068014. doi:10.1158/1078-0432.CCR-05-0632

11. Thomson S, Buck E, Petti F et al (2005) Epithelial to mesenchymal transition is a determinant of sensitivity of non-small-cell lung carcinoma cell lines and xenografts to epidermal growth factor receptor inhibition. Cancer Res 65(20):9455-9462. doi: 10.1158/0008-5472.CAN-05-1058

12. Witta SE, Gemmill RM, Hirsch FR et al (2006) Restoring E-cadherin expression increases sensitivity to epidermal growth factor receptor inhibitors in lung cancer cell lines. Cancer Res 66(2):944-950. doi:10.1158/0008-5472.CAN-05-1988

13. Yauch RL, Januario T, Eberhard DA et al (2005) Epithelial versus mesenchymal phenotype determines in vitro sensitivity and predicts clinical activity of erlotinib in lung cancer patients. Clin Cancer Res 11(241):8686-8698. doi:10.1158/1078-0432. CCR-05-1492

14. Buck E, Eyzaguirre A, Barr S et al (2007) Loss of homotypic cell adhesion by epithelial-mesenchymal transition or mutation limits sensitivity to epidermal growth factor receptor inhibition. Mol Cancer Ther 6(2):532-541. doi:10.1158/1535-7163.MCT-06-0462

15. Shrader M, Pino MS, Brown G et al (2007) Molecular correlates of gefitinib responsiveness in human bladder cancer cells. Mol Cancer Ther 6(1):277-285. doi:10.1158/1535-7163.MCT-06-0513

16. Frederick BA, Helfrich BA, Coldren CD et al (2007) Epithelial to mesenchymal transition predicts gefitinib resistance in cell lines of head and neck squamous cell carcinoma and non-small cell lung carcinoma. Mol Cancer Ther 6(6):1683-1691. doi: 10.1158/1535-7163.MCT-07-0138

17. Chakravarti A, Loeffler JS, Dyson NJ (2002) Insulin-like growth factor receptor I mediates resistance to anti-epidermal growth factor receptor therapy in primary human glioblastoma cells through continued activation of phosphoinositide 3-kinase signaling. Cancer Res 62(1):200-207

18. Jones HE, Goddard L, Gee JM et al (2004) Insulin-like growth factor-I receptor signalling and acquired resistance to gefitinib (ZD1839; Iressa) in human breast and prostate cancer cells. Endocr Relat Cancer 11(4):793-814. doi:10.1677/erc.1.00799

19. Engelman JA, Zejnullahu K, Mitsudomi T et al (2007) MET amplification leads to gefitinib resistance in lung cancer by activating ERBB3 signaling. Science 316(5827):1039-1043. doi: 10.1126/science. 1141478

20. Abdolzade-Bavil A, Hayes S, Goretzki L et al (2004) Convenient and versatile subcellular extraction procedure, that facilitates classical protein expression profiling and functional protein analysis. Proteomics 4(5):1397-1405. doi:10.1002/pmic.200300710

21. Thelemann A, Petti F, Griffin G et al (2005) Phosphotyrosine signaling networks in epidermal growth factor receptor overexpressing squamous carcinoma cells. Mol Cell Proteomics 4(4):356-376. doi:10.1074/mcp.M400118-MCP200

22. Petti F, Thelemann A, Kahler J et al (2005) Temporal quantitation of mutant Kit tyrosine kinase signaling attenuated by a novel thiophene kinase inhibitor OSI-930. Mol Cancer Ther 4(8):11861197. doi:10.1158/1535-7163.MCT-05-0114

23. Ross PL, Huang YN, Marchese JN et al (2004) Multiplexed protein quantitation in Saccharomyces cerevisiae using aminereactive isobaric tagging reagents. Mol Cell Proteomics 3(12):1154-1169. doi:10.1074/mcp.M400129-MCP200

24. Shilov IV, Seymour SL, Patel AA et al (2007) The Paragon Algorithm: a next generation search engine that uses sequence temperature values and feature probabilities to identify peptides from tandem mass spectra. Mol Cell Proteomics 6(9):1638-1655 
25. Bradshaw RA, Burlingame AL, Carr S et al (2006) Reporting protein identification data: the next generation of guidelines. Mol Cell Proteomics 5(5):787-788. doi:10.1074/mcp.E600005-MCP200

26. Buck E, Eyzaguirre A, Haley JD et al (2006) Inactivation of Akt by the epidermal growth factor receptor inhibitor erlotinib is mediated by HER-3 in pancreatic and colorectal tumor cell lines and contributes to erlotinib sensitivity. Mol Cancer Ther 5(8):2051-2059. doi:10.1158/1535-7163.MCT-06-0007

27. Heldin CH, Westermark B, Wasteson A (1981) Specific receptors for platelet-derived growth factor on cells derived from connective tissue and glia. Proc Natl Acad Sci USA 78(6):3664-3668. doi:10.1073/pnas.78.6.3664

28. Garton AJ, Crew AP, Franklin M et al (2006) OSI-930: a novel selective inhibitor of Kit and kinase insert domain receptor tyrosine kinases with antitumor activity in mouse xenograft models. Cancer Res 66(2):1015-1024. doi:10.1158/0008-5472.CAN-05-2873

29. Roberts WG, Whalen PM, Soderstrom E et al (2005) Antiangiogenic and antitumor activity of a selective PDGFR tyrosine kinase inhibitor, CP-673, 451. Cancer Res 65(3):957-966

30. Brown KA, Aakre ME, Gorska AE et al (2004) Induction by transforming growth factor-beta1 of epithelial to mesenchymal transition is a rare event in vitro. Breast Cancer Res 6(3):R215R231. doi:10.1186/bcr778

31. Peinado H, Olmeda D, Cano A (2007) Snail, Zeb and bHLH factors in tumour progression: an alliance against the epithelial phenotype? Nat Rev Cancer 7(6):415-428. doi:10.1038/nrc2131

32. Sheehan KM, Gulmann C, Eichler GS et al (2007) Signal pathway profiling of epithelial and stromal compartments of colonic carcinoma reveals epithelial-mesenchymal transition. Oncogene 27(3):323-331

33. Vega S, Morales AV, Ocana OH et al (2004) Snail blocks the cell cycle and confers resistance to cell death. Genes Dev 18(10):1131-1143. doi:10.1101/gad.294104

34. Zhang X, Wang Q, Ling MT et al (2007) Anti-apoptotic role of TWIST and its association with Akt pathway in mediating taxol resistance in nasopharyngeal carcinoma cells. Int $\mathrm{J}$ Cancer 120(9):1891-1898. doi:10.1002/ijc.22489

35. Lu Z, Ghosh S, Wang Z et al (2003) Downregulation of caveolin1 function by EGF leads to the loss of E-cadherin, increased transcriptional activity of beta-catenin, and enhanced tumor cell invasion. Cancer Cell 4(6):499-515. doi:10.1016/S15356108(03)00304-0

36. Grotegut S, von Schweinitz D, Christofori G et al (2006) Hepatocyte growth factor induces cell scattering through MAPK/Egr- 1-mediated upregulation of Snail. EMBO J 25(15):3534-3545. doi:10.1038/sj.emboj.7601213

37. Rikova K, Guo A, Zeng Q et al (2007) Global survey of phosphotyrosine signaling identifies oncogenic kinases in lung cancer. Cell 131(6):1190-1203. doi:10.1016/j.cell.2007.11.025

38. Jechlinger M, Grunert S, Tamir IH et al (2003) Expression profiling of epithelial plasticity in tumor progression. Oncogene 22(46):7155-7169. doi:10.1038/sj.onc. 1206887

39. Jechlinger M, Sommer A, Moriggl R et al (2006) Autocrine PDGFR signaling promotes mammary cancer metastasis. J Clin Invest 116(6):1561-1570. doi:10.1172/JCI24652

40. Strutz F, Zeisberg M, Ziyadeh FN et al (2002) Role of basic fibroblast growth factor-2 in epithelial-mesenchymal transformation. Kidney Int 61(5):1714-1728. doi:10.1046/j.15231755.2002.00333.x

41. Billottet C, Elkhatib N, Thiery JP et al (2004) Targets of fibroblast growth factor 1 (FGF-1) and FGF-2 signaling involved in the invasive and tumorigenic behavior of carcinoma cells. Mol Biol Cell 15(10):4725-4734. doi:10.1091/mbc.E04-04-0336

42. Suyama K, Shapiro I, Guttman M et al (2002) A signaling pathway leading to metastasis is controlled by $\mathrm{N}$-cadherin and the FGF receptor. Cancer Cell 2(4):301-314. doi:10.1016/S15356108(02)00150-2

43. Yamamoto N, Mammadova G, Song RX et al (2006) Tyrosine phosphorylation of p145met mediated by EGFR and Src is required for serum-independent survival of human bladder carcinoma cells. J Cell Sci 119(Pt 22):4623-4633. doi:10.1242/ jcs.03236

44. Morgillo F, Woo JK, Kim ES et al (2006) Heterodimerization of insulin-like growth factor receptor/epidermal growth factor receptor and induction of survivin expression counteract the antitumor action of erlotinib. Cancer Res 66(20):10100-10111. doi:10.1158/0008-5472.CAN-06-1684

45. Feng Q, Baird D, Peng X et al (2006) Cool-1 functions as an essential regulatory node for EGF receptor- and Src-mediated cell growth. Nat Cell Biol 8(9):945-956. doi:10.1038/ncb1453

46. Hlubek F, Brabletz T, Budczies J et al (2007) Heterogeneous expression of $\mathrm{Wnt} /$ beta-catenin target genes within colorectal cancer. Int J Cancer 121(9):1941-1948. doi:10.1002/ijc.22916

47. Moody SE, Perez D, Pan TC et al (2005) The transcriptional repressor Snail promotes mammary tumor recurrence. Cancer Cell 8(3):197-209. doi:10.1016/j.ccr.2005.07.009 